Communications in Physics, Vol.21, No. 2 (2011), pp. 153-159

\title{
PREPARATION AND INVERSTIGATION OF MEH-PPV FILMS USED FOR WHITE LIGHT EMITTING DIODES
}

\author{
NGUYEN NANG DINH, DO NGOC CHUNG, AND NGUYEN PHUONG HOAI NAM \\ University of Engineering and Technology, Vietnam National University, Hanoi \\ PHAM HONG DUONG \\ Institute of Materials Science, VAST
}

\begin{abstract}
With the aim to prepare white Light Emitting Diode (WLED), the conjugate polymer films like (Poly[2-methoxy-5-(2'-ethyl-hexyloxy)-1,4-phenylene vinylene] (MEH-PPV) were investigated. Spectroscopic (absorption and emission) spectra of the MEH-PPV films showed that this polymer is suitable for casting onto the chips of the blue InGaN LED to make WLED. The luminous flux measured on the WLEDs in the integrating sphere proved that the white light emission can be obtained from the combination of inorganic LED and conjugate (MEH-PPV) polymers with an optimal thickness and a high quality. The aging process of MEH-PPV films was found to be strongly dependent post-treatment conditions. Reasonable heat treatment condition for the $M E H$ $P P V$ polymers was suggested as in vacuum of $5 \times 10^{-2} \mathrm{~Pa}$ at a temperature of $120^{\circ} \mathrm{C}$ in , for 2 hous.
\end{abstract}

\section{INTRODUCTION}

Research and applications of solid state lighting have grown exponentially in the world since the discovery of InGaN fabrication process by Nakamura [1]. The rapid development of white light-emitting diodes (WLED) over the last few years has opened new opportunities in the general illumination market $[2,3]$. The efficiency of commercialized WLEDs is now around $80 \mathrm{~lm} / \mathrm{W}$, which is comparable with that of fluorescent lamps. There are several approaches to fabricate white light LED, for instance by using a blue or UV LED to excite some phosphors to give white light [4]. The ability to obtain different color correlated temperature (CCT) and high color rendering index (CRI) of WLED gives new features to this kind of light sources [2]. The other attempt can be considered, using conjugate polymers like Poly[2-methoxy-5-(2'-ethyl-hexyloxy)-1,4-phenylene vinylene] (MEH-PPV) to be covered onto a blue LED chip. It is known that MEH-PPV has the absorption peak at ca. $480 \mathrm{~nm}$ and emits yellow light with wavelength of ca. $580 \mathrm{~nm}$ [5], one can expect that under excitation of light of the inorganic LED with wavelength around $480 \mathrm{~nm}$, the blue-yellow combination light going out from the LED and MEH-PPV would be white light.

The aim of this work is to characterize properties of the MEH-PPV and the luminous flux of WLED made by covering the MEH-PPV layers onto blue LED chips. The color coodinates of WLED light was presented. 


\section{EXPERIMENTAL}

To characterize photoluminescent behavior of MEH-PPV films, we used a spincoating technique for depositing the films onto glass substrates. The substrates were ultrasonically cleaned in distilled water, followed by cleaning in ethanol and acetone. To deposit the polymer films onto glass, MEH-PPV solutions were prepared by dissolving MEH-PPV powder (produce of Alphadrich Ltd.) in xylene with a ratio of $10 \mathrm{mg}$ of MEHPPV in $1 \mathrm{ml}$ of xylene. The conditions for spin-coating are as follows: a delay time of 120 $\mathrm{s}$, a rest time of $30 \mathrm{~s}$, a spin speed of 1500 to $3000 \mathrm{rpm}$, an acceleration of $500 \mathrm{rpm}$ and finally a drying time of $2 \mathrm{~min}$. The thickness of the polymer layers was controlled by both the spin speed and solution content, the most suitable thickness for the absorption and photoluminescence characterization was found to be of $200 \mathrm{~nm}$, corresponding to a spin speed of $2400 \mathrm{rpm}$. All the samples were put in a fore vacuum box until the measurements.

The blue InGaN based LED chip used in our experiment was made by OptoGaN Group (Germany). The LED structure consists of a 30-nm-thick GaN buffer layer grown on $100 \mu \mathrm{m}$-thick sapphire substrate, a $1.5 \mu \mathrm{m}$-thick layer of undoped GaN, a $4.5 \mu \mathrm{m}$-thick layer of n-type GaN:Si, multiple quantum well (MQW) active layer, a $20 \mathrm{~nm}$-thick p-type $\mathrm{Al}_{0.2} \mathrm{Ga}_{0.8} \mathrm{~N}: \mathrm{Mg}$ layer and a $0.2-\mu$ m-thick p-type GaN:Mg layer. The MQW active layer of the blue LED consists of six 3 -nm-thick undoped $\mathrm{In}_{0.3} \mathrm{Ga}_{0.7} \mathrm{~N}$ well layers separated by 25 nm-thick undoped GaN barrier layers. The peak wavelength of this blue LED is 460 $\mathrm{nm}[2]$. The purchased wafer was cut into individual dies, delivered on medium tack blue tape.

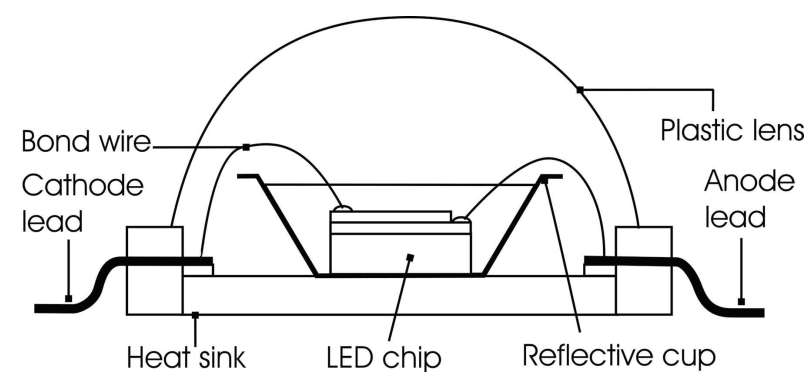

Fig. 1. Schematic illustration of the white LED structure. Basically a LED consists of four main components such as head sink, reflective cup, LED chip and plastic lens.

We use $1 \mathrm{~W}$ power blue LED for the study, with the die size around $1100 \mu \mathrm{m} \mathrm{x}$ $1100 \mu \mathrm{m}$ (Fig. 1). The die was then bonded on the heat sink using silver epoxy glue. The quality of thermal resistance between the LED die and the substrate determined the working temperature of the LED chip. The gold wires was bonded on electrode pads by using digital thermosonic multipurpose wire bonder model 626. Four gold wires (diameter $20 \mu \mathrm{m}$ ) are used for power LED in order to increase the heat dissipation. In difference from spin-coating MEH-PPV samples for the spectroscopic characterization, to make WLEDs, MEH-PPV solution with a larger concentration of polymer was prepared, then casted onto the surface area between the top of the LED chip and the lens (see Fig. 1). For casting 
the MEH-PPV layers, the pure MEH-PPV powder was dissolved in xylene with a ratio of $30 \mathrm{mg}$ of MEH-PPV in $1 \mathrm{ml}$ of xylene. After casting, the samples were annealed at $120^{\circ} \mathrm{C}$ for 2 hours both in air and in vacuum. The thickness of MEH-PPV films was controlled by the dilution content and the number of casting cycles, it was in the range from 200 $\mathrm{nm}$ to $1000 \mathrm{~nm}$. The electrical power used for lighting WLEDs was a DC current ranging from 1 to $350 \mathrm{~mA}$.

The absorption spectra were carried out on a Jasco UV-VIS-NIR V570. Photoluminescence (PL) spectra were carried-out by using a high resolution spectrometer Model Microspec-235b. The luminous flux of the WLED was measured by using a PMS-50 (PLUS) UV-VIS-NIR spectrophotocolorimeter.

\section{RESULTS AND DISCUSSION}

The absorption and photoluminescence spectra of MEH-PPV film were plotted in Fig 2. From this figure one can see that the MEH-PPV film has an absorption peak a 480 $\mathrm{nm}$ and two emission peaks at $570 \mathrm{~nm}$ and $640 \mathrm{~nm}$. This is consistent with the reporting results on MEH-PPV films deposited by spin-coating for preparation of OLED [6]. Thus a blue LED chip can be used for exciting luminescence of the MEH-PPV film.

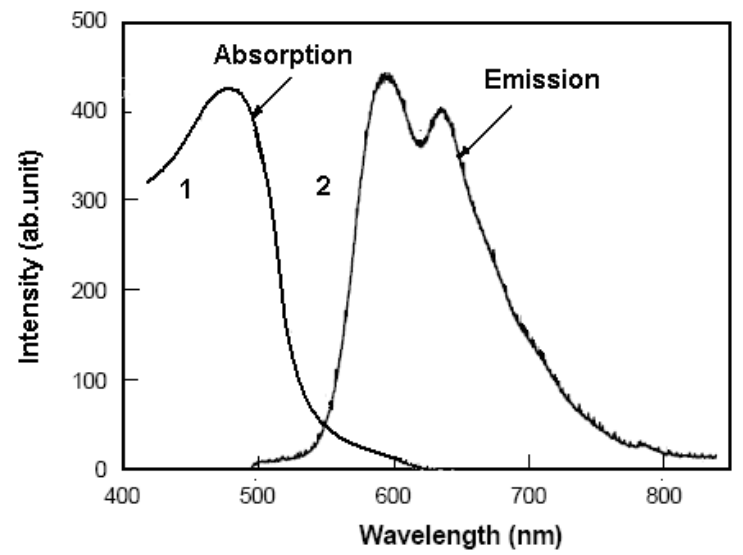

Fig. 2. Absorption (1) and emission (2) spectra of the MEH-PPV film. The thickness $d=200 \mathrm{~nm}$.

Fig. 3 presents plots of the PL spectra of MEH-PPV films with different film thickness and under an excitation of laser beam of $442 \mathrm{~nm}$. All the PL spectra for the MEHPPV films have two broad peaks respectively at $590 \mathrm{~nm}$ and $645 \mathrm{~nm}$. The peak observed at $590 \mathrm{~nm}$ is much larger than the one at $645 \mathrm{~nm}$, similarly to the electroluminescence spectra plotted in [7]. This shows that the commercial MEH-PPV polymer exhibits a large color range from yellow to red. Moreover, the intensity of the PL spectra is strongly dependent on the thickness of the spin-coated films. Approximatively, the ratio of the intensities of PL spectra of the samples having thicknesses of 200, 400 and $1000 \mathrm{~nm}$ is of 1:2:6. This result demonstrated that the thicker films the larger PL intensity was obtained. However, 


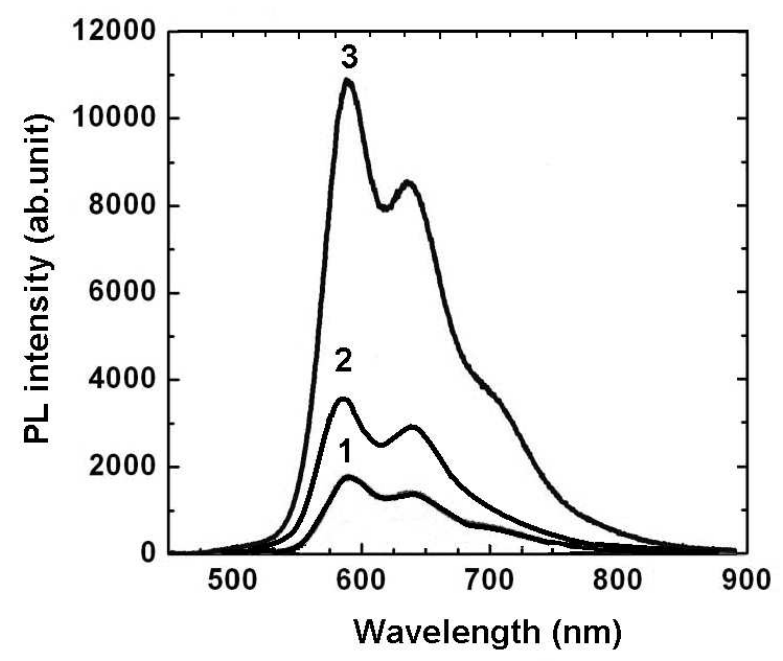

Fig. 3. Photoluminescence spectra of MEH-PPV films vs. the thickness (d) of the film: "1": $d=200 \mathrm{~nm}$, "2": $d=400 \mathrm{~nm}$ and "3": $d=1000 \mathrm{~nm}$.

the investigation of their morphological properties by SEM showed that uniform and wellsolidified films were obtained, with good adherence and a critical thickness, which is not larger than $400 \mathrm{~nm}$. In case of casting the thicker films, a large density of cracks on the films was observed. Therefore, for further investigation, we controlled the experimental conditions to cover MEH-PPV films with the critical thickness.

The luminous flux of the white LED was measured by using the integrating sphere in the PMS-50 (PLUS) system. Under an applied voltage of $2 \mathrm{~V}$ (with a $200 \mathrm{~mA}$ current), for a thin MEH-PPV film (about $200 \mathrm{~nm}$ ) that was casted on the LED chip, the emission light exhibited slight blue color. The results of the measured luminous flux is shown in Fig. 4a. The color coordinate corresponds to $x=0.1676$ and $y=0.0750$. This proves that the emission light from the LED went through-out without or very weakly exciting the MEH-PPV film. By increasing the thickness of MEH-PPV films up to $400 \mathrm{~nm}$, one can obtain the emission approaching to white light (Fig. 4b). The color coordinate is shown in Fig. 4b corresponds to $x=0.3471$ and $y=0.1730$.

The figure 5 shows an image made from a lighting WLED, the supply voltage and the current was, respectively $2 \mathrm{~V}$ and $200 \mathrm{~mA}$. One problem observed in this WLED is that the lasting time is still short. Within few minute, the yellow band was quenched and the original color of MEH-PPV was bleached. The origin of bleaching of film color and the quenching of the PL yellow band was suggested to be due to strongly heating polymer and the structure decomposition under blue light irradiation. In order to study the effect of quenching process of MEH-PPV film we used the He-Cd laser beam $(\lambda=442 \mathrm{~nm})$ served as excitation for the polymer luminescence. The PL spectra were recorded each $30 \mathrm{~s}$ by a cooled CCD, keeping the sample exposed continuously to the $200 \mathrm{~mW}$ blue laser beam. The laser spot diameter is around $1 \mathrm{~mm}$, corresponding to a power density is ca. 


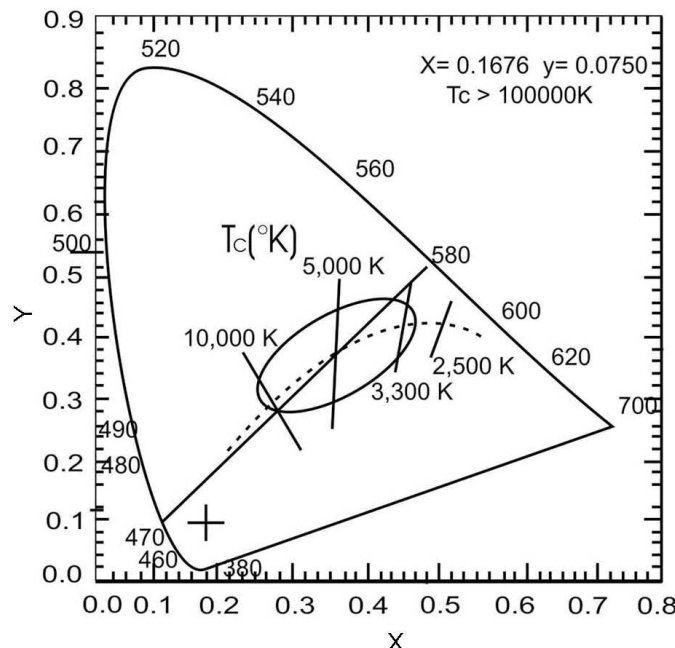

(a)

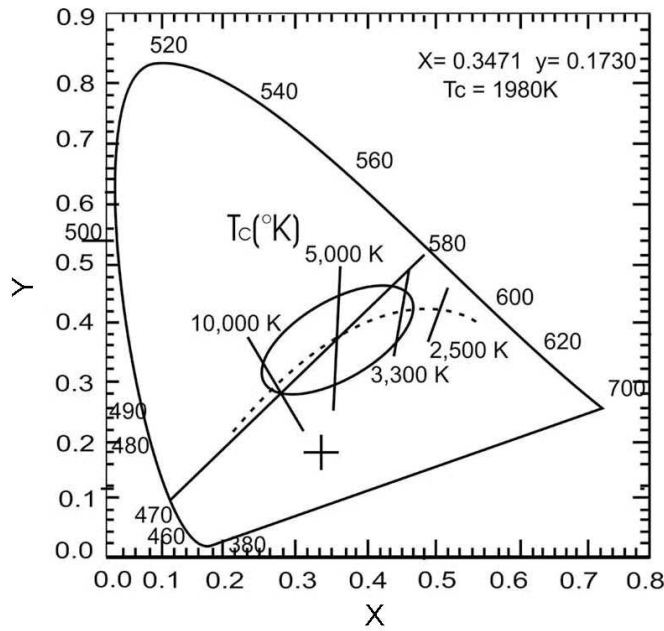

(b)

Fig. 4. Color coordinate of LED chip coated by a $200 \mathrm{~nm}$ (a) and $400 \mathrm{~nm}$ (b) thick MEH-PPV films.

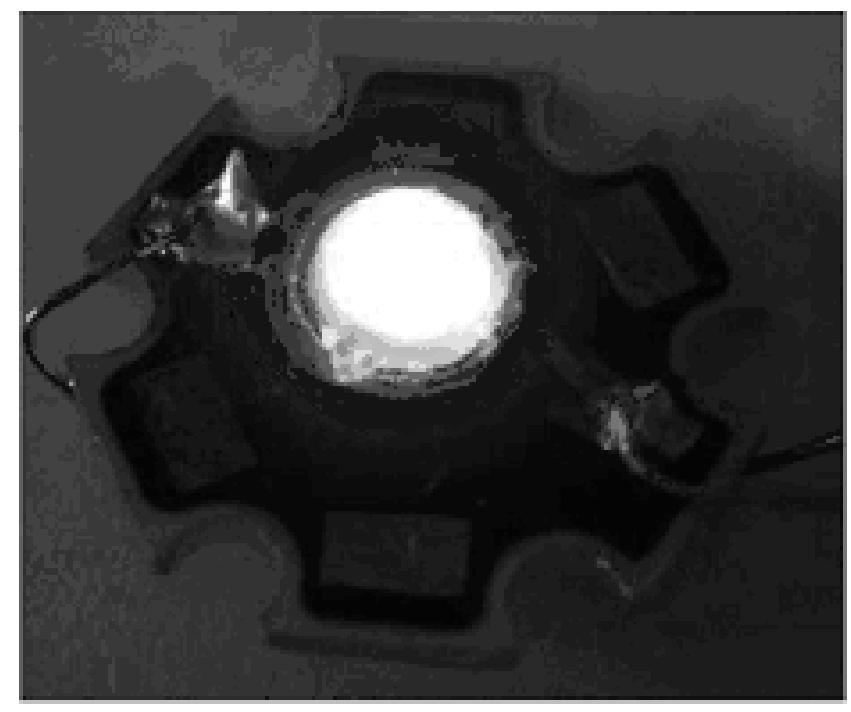

Fig. 5. Picture of a lighting WLED made by covering a $400 \mathrm{~nm}$ thick MEH-PPV layer.

$10 \mathrm{~W} / \mathrm{cm}^{2}$ ). Fig. 6 presents the plots of the dependence of PL intensity on the exposing time for the samples which were annealed in air and in vacuum at temperature of $120{ }^{\circ} \mathrm{C}$, for $2 \mathrm{~h}$.

From this figure one can see that after 30 minute, for the sample annealed in air, the PL intensity decreased more than $12 \%$ and it seemed to be decreasing more. This 


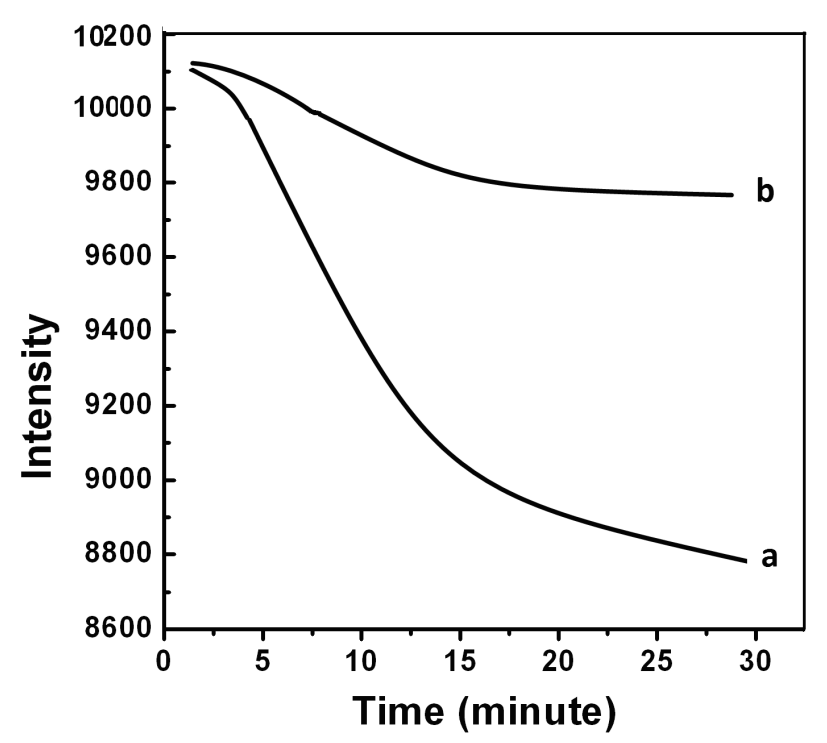

Fig. 6. The decrease in the PL intensity by exposing time of MEH-PPV films (thickness $\mathrm{d}=400 \mathrm{~nm}$ ) annealed in air (a) and in vacuum of $\sim 5 \times 10^{-2} \mathrm{~Pa}(\mathrm{~b})$.

film is ageing fast. For the sample annealed in vacuum, the aging occurred in first 15 min exposition, then reached a saturation state. The PL intensity decreased with a considerably small value, i.e. about $0.5 \%$. This demonstrates that quality of the polymers, for MEH-PPV in particular, was strongly affected by the oxygen in the air.

\section{CONCLUSIONS}

Optical properties of the conjugate polymer as MEH-PPV made by spin-coating with a critical thickness of $400 \mathrm{~nm}$ was characterized by absorption and photoluminescence spectroscopy. The results demonstrated that this polymer is suitable for making white light LEDs (WLED). The last was done by casting the MEH-PPV onto the chips of InGaN LEDs. The luminous flux measured on WLEDs in the integrating sphere showed the white light emission was obtained from the combination of inorganic LED and conjugate (MEHPPV) polymers with an optimal thickness and a high quality. The aging process of MEHPPV films was found to be strongly dependent post-treatment conditions. Reasonable heat treatment conditions for the MEH-PPV polymers was suggested as in vacuum of $5 \times 10^{-2} \mathrm{~Pa}$ at a temperature of $120^{\circ} \mathrm{C}$ in, for 2 hour.

\section{ACKNOWLEDGEMENTS}

This work was supported in part by the Protocol for the Scientific Cooperation between UET-VNU and PNU (South Korea) in the period of 2009 - 2010 and by the NAFOSTED in 2010 (Project Code: 103.02.88.09). 


\section{REFERENCES}

[1] S. Nakamura, T. Mukai and M. Senoh, Appl. Phys. Lett. 64 (1994) 1687.

[2] M. Yamada, T. Mitani, Y. Narukawa, S. Shioji, I. Niki, S. Sonobe, K. Deguchi, M. Sano, T. Mukai, Jpn. J. Appl. Phys. 41 (2002) L1431.

[3] N.-C. Chen, C.-M. Lin, Y.-K. Yang, C. Shen, T.-W. Wangi, M.-C. Wu, Jpn. J. Appl. Phys. 47 (2008) 8779.

[4] F. J. P. Schuurmans and M. D. Pashley, Light (IEEE J. on Selected Topics in Quantum Electronics) 8, No. 2 (2002) 333.

[5] Y.-T. Lin, T.-W. Zeng, W.-Z.Lai, C.-W. Chen, Y.-Y. Lin,Y.-S. Chang, W.-F. Su, Nanotechnology 17 (2006) 5781.

[6] Nguyen Nang Dinh, Le Ha Chi, Tran Thi Chung Thuy, Tran Quang Trung, and Vo-Van Truong, J. Appl. Phys. 105 (2009) 093518.

[7] S. A. Carter, J. C. Scott, J. Brock, J. Appl. Phys. 71(9) (1997) 1145.

Received 04 April 2010. 